\title{
Functional Anatomy of Essential Tremor: Lessons from Neuroimaging
}

R. Klaming and J. Annese

\section{ABSTRACT}

SUMMARY: The neuropathogenetic processes underlying essential tremor appear to cause subtle morphologic changes in neural networks that include multiple brain structures, primarily the cerebellum, brain stem, frontal lobes, and thalamus. One of the main challenges of neuroimaging in essential tremor is differentiating disease-specific markers from the spectrum of structural changes that occur due to aging. This review discusses recent neuroimaging studies in the light of current knowledge of the neuropsychology and pathology of the disease. We suggest that the application of multiple macroscopic and microscopic neuroimaging modalities, combined with personalized information relative to cognitive and behavioral symptoms, is the prerequisite for a comprehensive classification and correct diagnosis of essential tremor.

ABBREVIATIONS: $\mathrm{ET}=$ essential tremor

$\mathbf{E}_{\mathrm{d}}$ ssential tremor (ET) is one of the most common yet least understood movement disorders. ${ }^{1}$ It is primarily characterized by a shaking of the hands and forearms that is evident during simple voluntary actions, such as drinking, pouring, eating, or writing. ${ }^{2}$ For this reason, in addition to causing obvious disruption of daily activities, patients with ET often avoid social situations, even though they develop strategies to cope with the condition. Tremor is not limited to the upper limbs; the head, neck, voice, legs, or trunk may also be affected. Although ET is much more common in the elderly, the tremors can appear in children and young adults. Therefore, it cannot be considered purely an age-related disease, nor is it clearly sex-specific.

Despite the serious impairment that most patients experience, ET was, until recently, viewed merely as a benign, monosymptomatic (ie, motor) condition. Increased public awareness and new research have shifted the perception of ET to that of an actual neurodegenerative disease that is characterized by motor symptoms and cognitive

From The Brain Observatory, San Diego, California; and Department of Radiology, University of California, San Diego, San Diego, California.

J.A. and R.K. were partially supported by 2 research grants from the National Eye Institute, R01 EY018359-02 and ARRA R01 EY018359-02S1 (Jacopo Annese, Principal Investigator) and a research grant from the National Institute for Mental Health, R01 MH084756 (Jacopo Annese, Principal Investigator). The work was also supported, in part, by a commitment from the Vice Chancellor of Health Sciences at the University of California, San Diego and by Clinton and Margaret Spangler's generous financial donation.

Please address correspondence to Jacopo Annese, PhD, The Brain Observatory, 3510 Dunhill St, San Diego, CA 92121; e-mail: jannese@ucsd.edu

- Indicates open access to non-subscribers at www.ajnr.org

http://dx.doi.org/10.3174/ajnr.A3586 changes. ${ }^{3}$ Nevertheless, unlike other neurologic conditions such as Alzheimer and Parkinson diseases, for which the dynamics and the topography of degeneration are reasonably well-mapped, the neuropathologic phenomena underlying ET seem to be much more subtle and their distribution, more elusive.

To date, specific noninvasive markers of ET have not been identified and localized. This may be because the pathology of ET is overshadowed by broad features of age-related neurologic degeneration. It is also possible that available tools do not afford the resolution (Fig 1) or scope of analysis to detect patterns of correlation that may constitute the neural architecture of the disease.

\section{The Diagnosis of ET Is Not Definitive}

Given that definitive radiologic and pathologic criteria do not exist, ${ }^{2,4}$ the diagnosis of ET is based solely on behavioral symptoms. Relevant factors to establish a diagnosis and distinguish ET from other movement disorders include the age of onset, the evolution of clinical signs with time, which factors aggravate the symptoms, past or chronic exposure to toxic substances (heavy metals in particular), and family history.

In the case of other neurodegenerative disorders, the diagnosis of the disease can be validated at postmortem examination. For example, postmortem gross dissection of the brain of patients with Parkinson disease reveals lack of pigmentation in the substantia nigra. Cortical thinning and enlargement of the ventricles are macroscopic indicators of Alzheimer disease pathology, which is ultimately confirmed when plaques and tangles are present in silver-stained histologic preparations. The distribution of Alzheimer disease and Parkinson disease pathology follows a very con- 

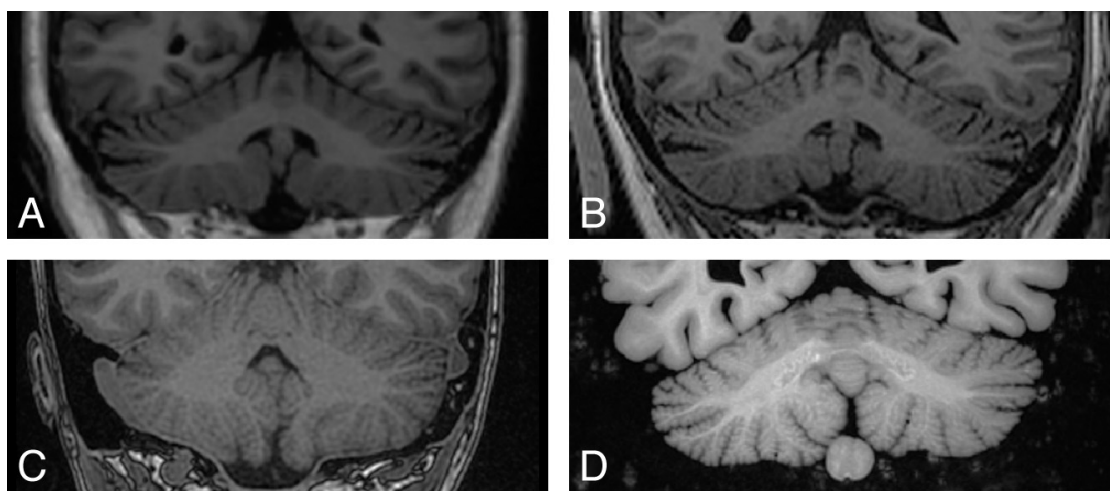

FIG 1. Comparison among images of the cerebellum obtained with different hardware and in different imaging conditions. In vivo 1.5T MR image $(A)$ of a patient with $E T$, in vivo $3 T$ MR image $(B)$ of the same patient, postmortem high-resolution 1.5T MR image $(C)$ acquired in situ, and 1.5T MR image $(D)$ of formaldehyde-fixed specimen obtained ex situ. The direct comparison between the modalities shows the morphologic detail intrinsic to the cerebellar cortex and the potential of different scans (and different scanners) to reveal geometric complexity and contrast between the cerebellar cortex and WM. A, Sagittal TT 3D inversion recovery fast-spoiled gradient recalled (FSPGR); matrix size, $256 \times 256$; pixel spacing, $1 / 1 \mathrm{~mm}$; section spacing, $1.2 \mathrm{~mm}$; section thickness, $1.2 \mathrm{~mm}$. B, Tl FSPGR; matrix size, $256 \times 256$; pixel spacing, isometric $1 \mathrm{~mm}$; section spacing, $1 \mathrm{~mm}$; section thickness, $1 \mathrm{~mm}$. C, High-resolution inversion recovery $\mathrm{T1}$ spoiled gradient recalled imaging; matrix size, $512 \times 512$; pixel spacing, $0.5273 \times 0.5273 \mathrm{~mm}$; section spacing, $0.7 \mathrm{~mm}$; section thickness, $0.7 \mathrm{~mm}$. D, High-resolution coronal T1 3D inversion recovery FSPGR; matrix size, $512 \times$ 512; pixel spacing, $0.5 \times 0.5 \mathrm{~mm}$; section spacing, $1 \mathrm{~mm}$; section thickness, $1 \mathrm{~mm}$.

sistent pattern, as described by the staging of Braak and Braak ${ }^{5}$ and Braak et al. ${ }^{6}$

For ET, there is no particular protocol for examination of the tissue at a microscopic level. Moreover, because ET seems to affect several structures in the brain, the typical sampling protocol (ie, the selection of only few small blocks of tissue) for institutional neuropathologic examination may not be adequate to map the distribution of cellular-level pathology. ${ }^{7}$ It is conceivable, however, that ET-specific patterns of the distribution of susceptible cell types may emerge as more brain specimens are examined postmortem. Hence, it is important to invest in the curation and study of collections of brain specimens donated by patients who were thoroughly characterized.

\section{Cognitive Deficits in ET}

As mentioned above, ET is not a purely motor disorder but has instead been linked to additional mild cognitive deficits. ${ }^{3}$ Despite an increasing body of neuropsychological studies of ET, the set of diagnostic criteria currently used does not include cognitive symptoms. ${ }^{2,4}$ The affected functions are fairly heterogeneous, including impairments in attention, executive function, verbal fluency, visuospatial functioning, memory, and working memory. ${ }^{3}$ A clear neuropsychological profile of ET is needed, and the pathophysiology of these behavioral symptoms remains to be established.

When patients with ET were asked to perform a task measuring selective attention and response inhibition ${ }^{8}$ as well as a verbal working memory task known to involve cerebellar circuits, ${ }^{9}$ their performance was comparable with that of controls. However, neural overactivation was detected, likely reflecting compensatory responses to subtle cognitive deficits. ${ }^{89}$ The question of whether this compensation fails as the disease progresses, which would imply a degenerative component, needs to be explored with longitudinal studies. ${ }^{9}$
In the context of neuropsychological symptoms, cerebellar disease and damage have been associated with a pattern of cognitive deficits similar to that observed in ET. ${ }^{10}$ The deficits seen in individuals with cerebellar lesions are believed to be produced by a disruption in the communication processes between the cerebellum and the prefrontal cortex via parietal, temporal, and limbic structures. ${ }^{10}$

\section{Diverse Etiologic Factors in ET}

As mentioned above, tremor onset and progression are important for diagnosing ET; however, its etiology has not been ascertained. The "usual suspects" in terms of risk factors are aging, ${ }^{11}$ specific environmental agents, ${ }^{12}$ and hereditary predisposition. ${ }^{13}$ Discrepancies between multisite genetic studies and the expression of diverse phenotypes in the same family suggest genetic heterogeneity. ${ }^{14}$ The logistics and the data to support large-scale neuroimaging studies combined with genetic studies to identify variants associated with putative noninvasive markers of ET are currently lacking. Therefore, the focus should be placed on developing accurate methodologies to identify neuroimaging markers and relate these to individual histories and etiologies.

Environmental factors discussed in the context of ET include increased blood levels of lead and beta-carboline alkaloids. ${ }^{12}$ These neurotoxins cause damage to the cerebellum and the olivary-cerebellar tract. ${ }^{15}$ Toxicologic research to evaluate accumulation of agents in the tissue from multiple brain structures could reveal buildup patterns in the brain of patients with ET. Whole-brain, noninvasive imaging techniques could also be used to relate neurodegeneration in the brain to other measures of toxin accumulation, such as lead poisoning. ${ }^{16}$

\section{Options and Reticence in Treatment}

The symptoms of ET are treated pharmacologically only when the tremor is severe enough to interfere significantly with the performance of daily activities. The 2 most commonly prescribed drugs are primidone, an anticonvulsant, and propranolol, a beta-adrenergic-blocking agent. Medication may be taken either on a daily basis or as needed, for instance, before a social event. Crucially, treatment is ineffective in $>30 \%$ of the patient population. ${ }^{17}$ These statistics are partly responsible for many patients not seeking or adhering to treatment unless the tremor is intolerable.

In the most severe and pharmacologically unresponsive cases, deep brain stimulation of the thalamus can be effective in subduing and even eliminating tremor. ET symptoms are reduced when the electrode is positioned within the ventral intermediate nucleus of the thalamus, ${ }^{18}$ a region that receives input from the striatum (specifically, the globus pallidus) and the cerebellum and, in turn, projects to the motor cortex. ${ }^{19}$ The fact that deep 
brain stimulation works suggests not only that multiple connected brain regions are involved in the production of the tremors but also that ET shares common neurologic substrates with Parkinson disease, for which deep brain stimulation is an established palliative procedure.

Not surprisingly, alcohol relieves tremor symptoms in many patients with $\mathrm{ET},{ }^{20}$ likely via the inhibition of glutamatergic pathways, but only temporarily. As the effects of ethanol wear off, the tremor symptoms actually worsen. Most interesting, transcranial magnetic stimulation, applied on the occipital area (in the region of the inion), has the same temporary mitigating effect as ethanol on tremor symptoms. ${ }^{21}$ Given the shallow range of the magnetic pulses in transcranial magnetic stimulation, unless the visual cortex is inexplicably implicated in the disease, we conclude that the cerebellum is at least partially involved in tremor and that the body of research that focused on this structure is vindicated.

\section{ET and the Aging Brain}

It is difficult (if not impractical) to distinguish ET-related pathology from more general age-related structural changes that are detectable with MR imaging. ET is more prevalent among the elderly, ${ }^{1}$ and neuroimaging must inevitably contend with this potential bias. If the onset of ET occurs later in life (ie, after 65 years of age), the progression of tremor symptoms is faster and more severe degenerative pathology in the cerebellum occurs. ${ }^{22}$ This is puzzling because one would expect a greater accumulation of degeneration at the cellular level in longer-standing early-onset cases.

From a cognitive perspective, ET has also been linked to a faster decline compared with healthy age-matched subjects. ${ }^{23}$ Furthermore, late-onset ET has been linked to an increased risk of dementia. ${ }^{11}$ The relationship between cognitive decline and tremor symptoms is unclear. However, including well-defined cohorts with ET in the context of large-scale longitudinal neuroimaging studies that also track cognitive functioning could identify anomalous rates of cognitive decline and patterns of cerebral atrophy as valuable predictive indicators of ET. To be revealed, these effects may require specialized imaging and analysis methods. As a start, given that many individuals with ET do not tend to volunteer information about their condition unless a diagnosis is made and the personnel who perform the scans are not trained (or instructed) to pay attention to the signs of ET, it would be useful to screen for ET in participants of abundant ongoing imaging studies. $^{24}$

\section{The Lack of a Definitive Neuropathologic Signature for ET} At least 2 separate histopathologic features have been observed postmortem in samples of brains of patients with ET: loss of Purkinje cells in the cerebellum (in two-thirds of cases examined) and the presence of Lewy bodies in the brain stem (these features have never been found in the same brain). ${ }^{25,26}$ One explanation for this scenario is that distinct abnormalities at the cellular level are markers of different subtypes of ET. ${ }^{27}$

Purkinje cells, which are motor in function, are inhibitory neurons; therefore, their loss could be linked to hyperactivity of the cerebellum and, consequently, to tremor symptoms. ${ }^{26}$ Lewy bodies concentrate in the locus coeruleus, ${ }^{25}$ which is the most important source for norepinephrine in the brain and has major efferent connections to Purkinje cells in the cerebellum. ${ }^{15}$ It has, therefore, been proposed that pathologic changes in the locus coeruleus may eventually lead to changes in Purkinje cells ${ }^{26}$ (but then we should find both features in the same brain). Lewy bodies are prevalent in tissue from many elderly donors, including those without neurodegeneration or dementia in the last years of their lives; thus, it is unlikely that this pathology could ever become the hallmark for ET. ${ }^{28}$

"Torpedoes" are attenuated axonal swellings that are believed to be secondary markers related to the degeneration and death of Purkinje cells. ${ }^{27}$ "Torpedo " sounds certainly more rare and exotic, but a review of the literature published on these peculiar silver-stained structures gives the disappointing result that they are indeed not characteristic of normal brain aging and they are not unique to brains of patients with ET either. Finally, 2 histologic studies reported abnormal attenuation of tangled basket cells surrounding the Purkinje cell bodies ${ }^{29}$ and increased Bergman astrogliosis. ${ }^{27}$ The cause and effects of these secondary pathologic changes remain unclear.

The findings reported in this section originate largely from studies that re-examined the same collection of brain specimens ${ }^{28}$ (studies on the neuropathology of ET are still conducted by a handful of laboratories and on very few brains). Any apparent convergence of results could, therefore, be attributable, at least in part, to the paucity and redundancy of analyses. However, even within this small and contained research community, not everyone agrees with the idea that ET should be seen primarily as a cerebellar disease. $^{30,31}$ As mentioned earlier, there may very well be several subtypes of ET that display different (partly overlapping) pathology. It is possible that the samples studied so far only show a limited cross-section and incomplete picture of the disease. In either case, the logical solution is to increase collection and distribution of research samples for controlled (and reproducible) postmortem studies. Availability of biosamples for studies of the neuropathology of ET should improve both in terms of quantity but also in quality - that is, each sample should come fortified with clinical, neuropsychological, and even radiologic characterizations. $^{7}$

\section{The Need for Neuroimaging Markers as a Diagnostic Tool for ET}

Histologic studies can give a definitive view of the tissue-level phenomena underlying observed signs of neurologic disease, but unfortunately, they cannot assist directly with the premorbid detection of pathologic changes. MR imaging, on the contrary, has promise in terms of predicting and tracking clinical outcomes. Several MR imaging studies, published in the past decade, sought to detect ET-specific morphologic abnormalities. Most studies highlighted atrophic changes in different lobules of the cerebel$\operatorname{lum}^{32,33}$ (but also in other brain regions ${ }^{34,35}$ ).

Patients with both head and hand tremor showed reduced GM volume in the vermis of the cerebellum, compared with patients with only hand tremor. ${ }^{32}$ Increased GM and WM atrophy (relative to analogous measures in control subjects) was also detected in the frontal, occipital, parietal, and temporal lobes and the medulla and insula. ${ }^{34,35}$ Taken at face value, these reports suggest a 
generalized effect of ET on the cerebellothalamocortical network, ${ }^{34,35}$ but these positive results are not ubiquitous in the literature. ${ }^{36}$ The lack of definitive macroscopic markers for cerebellar atrophy ${ }^{36}$ and ET-specific morphologic changes reflect the ambiguous picture that currently describes pathology at the microscopic level.

Some questions regarding ET pathology could be explored with existing tools. For example, it should be possible to determine whether ET affects the entire cerebellum or whether it selectively affects specific lobules or nuclei of the cerebellum. ${ }^{37}$ This distinction may be of particular interest because the anterior lobule of the cerebellum is associated with motor function while the posterior regions are involved in cognition. ${ }^{37}$ In particular, neural activation patterns among the frontal regions and the cerebellar hemispheres and the dentate nucleus are involved in executive functioning and verbal working memory. ${ }^{38}$ Perhaps ET affects the anterior regions of the cerebellum first, leading to motor symptoms, and then spreads to the posterior regions as the disease progresses, leading to cognitive impairments.

Do patients with ET with more severe cognitive impairments also show more severe atrophy in the posterior lobules of the cerebellum? Remarkably, none of the structural MR imaging studies discussed in this review evaluated the level of cognitive impairment in their study participants. In addition to mapping the topography of structural abnormalities, defining the relative changes in different tissue compartments by using specialized imaging sequences and morphometric analyses could lead to identifying specific degeneration patterns in ET, distinguishable from those occurring due to aging.

Preliminary findings from a small ET pilot study have revealed selective atrophy in the cerebellar cortex in the absence of overall cortical atrophy. ${ }^{39}$ In particular, the sulci of the cerebellum were found to be deeper and wider, likely indicating selective deterioration of cerebellar GM. Most interesting, the patients showed no overall volume loss in the cerebellum and minimal cerebral cortical atrophy. In addition to neuroimaging, subjects also underwent detailed neuropsychological assessment and tremor quantification, which revealed different cognitive profiles and tremor characteristics. These preliminary findings suggest that cerebellar cortical atrophy may serve as a potential selective neuroimaging marker for ET, separate from the other effects of age-related degeneration. ${ }^{39}$

\section{Evaluating Neural Networks Rather than Localized Brain Regions}

Undoubtedly, evidence from most published neuroimaging studies suggests that the cerebellum or neural networks involving the cerebellum play a key role in the pathophysiology of ET. ${ }^{32-35,40-42}$ The unresolved question is whether the tremors originate in the cerebellum and are subsequently mediated by other structures within that network or whether a concerted disruption in connectivity between several brain structures causes ET-related symptoms.

MR-based diffusion imaging measures water diffusion and diffusion directionality along axons as an indicator of axonal injury. In ET research, diffusion imaging studies focus on fiber tracts projecting to and from the cerebellum. The first study to explore functional connectivity in patients with ET compared with healthy controls used DWI. ${ }^{43}$ No major structural abnormalities were detected in the pons, middle cerebellar peduncles, cerebellar WM, dentate nucleus, red nucleus, thalamus, caudate, putamen, pallidum, and frontal WM. ${ }^{43}$ Yet, DWI may not be sufficiently sensitive because it measures diffusion in only 1 direction as opposed to DTI, which measures diffusion in multiple directions. ${ }^{44}$ If that were the case in this study, more subtle WM abnormalities may have been overlooked in the ET cohort. ${ }^{43}$

Subsequent DTI studies explored WM fiber integrity and directionality in patients with ET. DTI measures mean diffusivity and ADC, as markers of the magnitude of water diffusion along fiber tracts, and fractional anisotropy, as a marker of the directionality of water diffusion. ${ }^{45}$ Decreased fractional anisotropy and increased mean diffusivity/ADC indicate WM damage. ${ }^{45}$ Increased ADC values of the red nucleus, indicative of neuronal loss or injury in this region, have been reported in patients with ET compared with healthy controls. ${ }^{44}$

Because it is possible that pathologic changes in ET may spread with time, affecting various brain regions, the red nucleus may be a structure affected earlier than others. ${ }^{44}$ However, this explanation is unlikely because abnormal DTI values in the red nucleus are not a consistent finding in patients with ET. ${ }^{40}$ Likewise, while longer disease duration did correlate with lower fractional anisotropy values in the dentate nucleus in 1 study, ${ }^{40}$ a relationship between disease duration or tremor severity and DTI measures was not confirmed by others. ${ }^{41,42}$ This discrepancy shows that the question of whether ET is progressive warrants further study.

That the tremor origin has not been localized in ET thus far suggests that an abnormal communication between several brain regions causes motor symptoms. Several neural loops have been proposed to be affected. For instance, DTI values of the superior cerebellar peduncle fiber tracts, which receive input from the dentate nucleus and send output to the red nucleus, indicated damaged WM in a study by Nicoletti et al. ${ }^{40}$ Based on these findings, a disturbance in the dentatorubro-olivary tract (ie, neural fibers connecting the dentate nucleus via the superior cerebellar peduncles with the red nucleus and inferior olive) may underlie tremor genesis in ET. ${ }^{40}$

A later study, however, failed to find WM abnormalities in the middle and superior cerebellar peduncles. ${ }^{41}$ Instead, compromised fiber integrity was found in the inferior cerebellar peduncles, suggesting a disturbance in tracts projecting through the cerebellum via the inferior cerebellar peduncles. ${ }^{41}$ Based on reduced fractional anisotropy in the pons, cerebellum, and midbrain and extensive WM damage in the frontal cortex and temporoparietal regions in patients with $\mathrm{ET}$, others proposed that an abnormality in the cerebellothalamocorticocerebellar loop is responsible for the tremor. ${ }^{46}$ Similarly, compromised WM has been reported in frontoparietal regions, cerebral hemispheres, the thalamus, and brain stem. ${ }^{42}$

In summary, with the exception of 1 study, ${ }^{43}$ most DTI research in ET demonstrated that widespread disintegrity of cerebral and cerebellar fiber tracts is common and likely underlies tremor generation. ${ }^{40-42,46}$ Although it is known that DTI measures are indicators for WM integrity, the exact nature underlying these pathologic processes is unknown. Whether demyelination, 
myelin injury, neuronal loss, or other processes underlie abnormal DTI values in patients with ET remains to be ascertained.

\section{Potential Bias in ET Neuroimaging Studies}

The obvious discrepancy between the results of different studies may be due to a number of methodologic inconsistencies. For example, while some studies used region-of-interest-based analysis methods, ${ }^{44}$ others used whole-brain analysis approaches ${ }^{46}$ or both. ${ }^{41}$ Region-of-interest-based analysis is more biased, and overall structural abnormalities may be overlooked. Whole-brain analysis, on the other hand, allows exploration of widespread networks but may not be specific enough, with the risk of ignoring smaller regions and nuclei. ${ }^{41}$

The data that make up all of the neuroimaging descriptions of ET were acquired at different magnet field strengths (most at $1.5 \mathrm{~T}^{32,33,36,40,46}$ and $\left.3 \mathrm{~T}^{34,35,41,42,44}\right)$. Accordingly, contrast and resolution, the parameters that, for the most part, guide the interpretation and analysis of the images, cannot be assumed constant. The cerebellum is a very complex structure in terms of geometry, and the scale of morphologic features (and their aberrations) is much smaller than that in the cerebral hemispheres. The fractal gyrification of the cerebellar cortex is an exquisite example of this principle. Cerebellar anatomy is extremely difficult to visualize at the relatively low resolutions afforded by clinical scans ${ }^{34}$ (Fig 1). The need for validation studies across scanners of different manufacturers and with different field strengths is even more crucial for ET than for other areas of investigation using MR imaging to model cerebral structures.

Other factors that varied considerably among studies are subject and tremor characteristics. Because it is likely that ET is a heterogeneous disease with different phenotypes, the general ET diagnosis could be inadequate to define cohorts undergoing imaging studies. It may be necessary to screen for more subtle differences between patients, such as the pattern of tremor manifestation (circadian fluctuations, effect of medication, and so forth), the history and severity of the disease (these could be documented via personalized medical history and could be measured quantitatively with commercially available tremormeters, respectively), and simple genetics (discriminating between familial-versusnonfamilial ET).

While some patient cohorts were limited to subjects with a family history of ET, ${ }^{32,40}$ others included patients with and without familial ET ${ }^{41-43,46}$ or did not control for this variable. ${ }^{44}$ While a positive family history is not the only factor proposed to play a role in the etiology of ET, this factor should be controlled for, because it could be a possible confounder. That ET often goes undiagnosed for many decades and subjects may not know about the medical history of estranged or deceased relatives should also be taken into consideration.

Finally, only 3 of the neuroimaging studies reviewed included young adults. ${ }^{35,42,46}$ The age bias toward elderly subjects, not only dictated by the prevalence of the disease among older men and women but also by the predominance of subjects who are retired and amenable to participate and adhere to the research, could overlook crucial clues deposited by ET in otherwise young and "clean" brains. Last, neuroimaging studies might be looking too selectively at structures that are known to be "motor," such as the cerebellum; the effect is made obvious in Fig 2.

\section{The Dysfunctional Correlates of ET}

Using PET, researchers have looked for metabolic abnormalities that may trigger the tremor, such as increased blood flow in the cerebellum of patients with ET (compared with control subjects). ${ }^{47,48}$ Patients with ET who underwent PET showed increased blood flow in the striatum, thalamus, and sensorimotor cortex ${ }^{47}$ and in the region of the red nucleus. ${ }^{48}$ These pontine structures were also implicated by measures of blood flow with fMRI. ${ }^{49}$ Bilateral activation of the red nucleus, along with the dentate nuclei and both cerebellar hemispheres, was observed in patients with ET, while control subjects only showed unilateral activation of these regions when they affected tremor with the opposite limb.

PET research has also provided a neurologic explanation for the above-mentioned fact that consumption of ethanol modulates ET. This effect was mirrored by decreased metabolic rates in the cerebellum and increased activation in the inferior olivary nuclei. ${ }^{47,20}$ Magnetoencephalography, which is characterized by a high temporal resolution, demonstrated coherent activation patterns between the arm muscle and motor cortices, thalamus, brain stem, and cerebellum, suggesting that this network of structures plays a role in ET-related tremor. ${ }^{50}$ However, such coherent activation patterns were not confirmed by others questioning the temporal dynamics of the disease. ${ }^{51}$

By measuring the level of various cellular metabolites, in particular NAA and total creatine, MR spectroscopy can localize neuronal injury or loss in the brain. Patients with ET showed reduced $\mathrm{NAA} /$ total creatine in the cerebellum, and these values correlated with both age and tremor severity, suggesting the degenerative nature of this disease. ${ }^{52}$ The readers of this review will be used to cautionary tales by now; therefore, they will understand that other studies could not confirm the above-stated relationship between metabolic abnormality, age, and tremor. ${ }^{53}$

Due to the poor spatial resolution afforded by MR spectroscopy in most typical clinical and research settings, it was possible to neither measure metabolic levels selectively in smaller nuclei nor distinguish between WM and GM within the cerebellum. ${ }^{52,53}$ In addition, longitudinal measurements in the same subject would also be required to link metabolic changes to the progression of tremor symptoms. ${ }^{52}$

\section{CONCLUSIONS}

Neuroimaging research in the field of ET has demonstrated that widespread structural changes in cortical and subcortical brain regions and metabolic abnormalities are common in symptomatic patients. Clearly, detecting structural and functional changes in the cerebellum is essential in understanding the neurologic basis of motor and behavioral manifestations of ET. Nevertheless, the detailed characterization of cerebellar abnormalities that can be obtained by applying multiple, noninvasive, and postmortem neuroimaging modalities (at macroscopic and microscopic resolution, respectively) needs to be integrated with a temporal and spatial network-based approach to trace the origin and progression of the symptoms. 


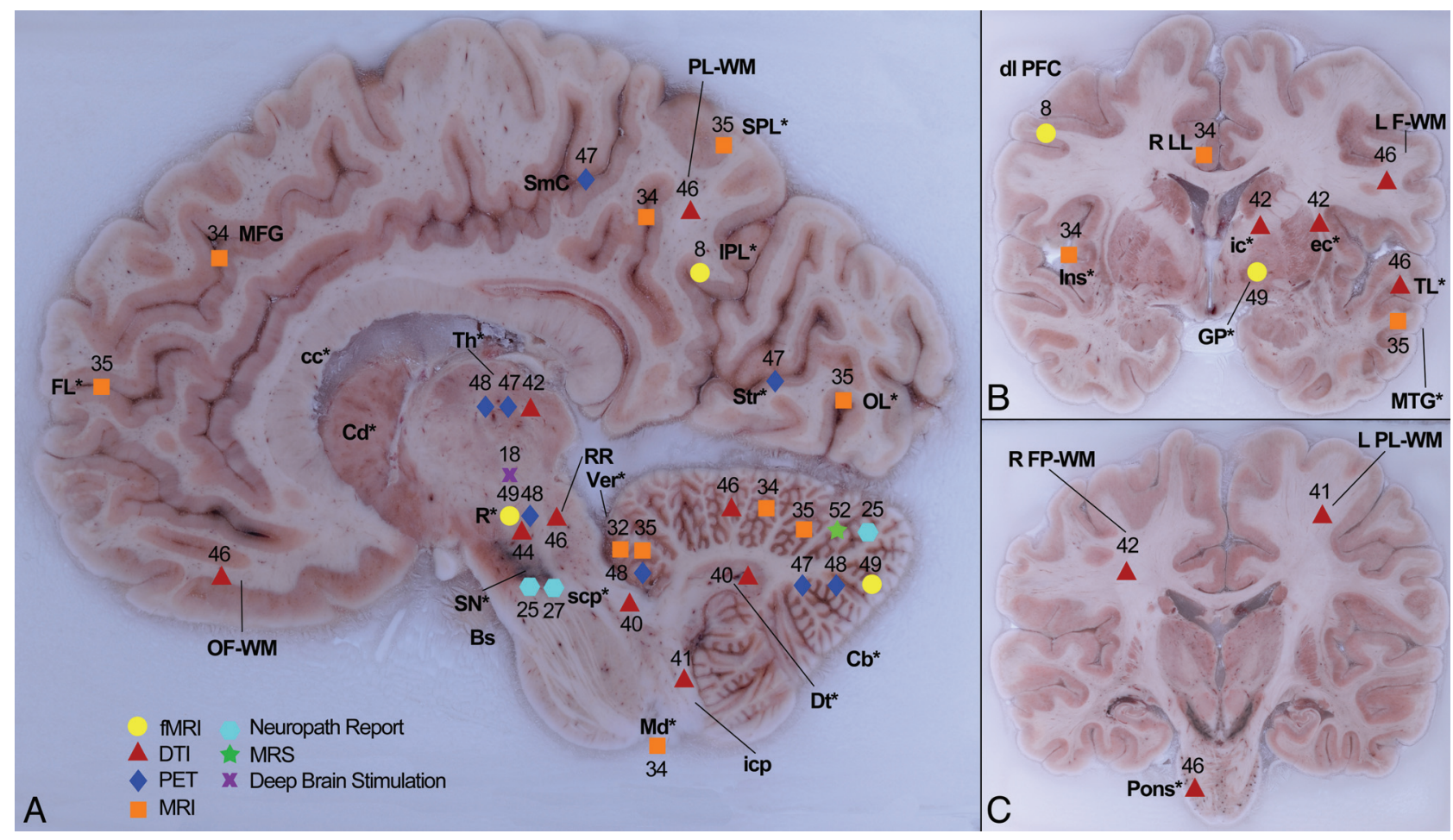

FIG 2. Summary of observed or measured phenomena derived from multiple neuroimaging modalities, neuropsychological testing, and postmortem examination (indicated by differently colored symbols). The location of neuroimaging results was projected onto tomographic anatomic images of the brain acquired during the sectioning process ${ }^{7}$ : Purkinje cell loss, ${ }^{25}$ presence of Lewy bodies, ${ }^{25,27}$ reduced fractional anisotropy, ${ }^{40,41,46}$ placing of deep brain stimulation electrode, ${ }^{18} \mathrm{GM}$ loss, ${ }^{32,34,35}$ increased blood flow, ${ }^{8,47,48,49}$ WM loss, ${ }^{34}$ increased ADC,${ }^{44}$ increased mean diffusivity, ${ }^{40,41,44}$ increased axial diffusivity, ${ }^{42}$ reduced NAA/total creatine. ${ }^{52}$ Bs indicates brain stem; $\mathrm{Cd}^{*}$, caudate; $\mathrm{Cb}^{*}$, cerebellum; $\mathrm{Cc}^{*}$, corpus callosum; dl PFC, dorsolateral prefrontal cortex; $\mathrm{Dt}^{\star}$, dentate nucleus; ec*, external capsule; FL*, frontal lobes; GP*, globus pallidus; icp*, inferior cerebellar peduncle; Ins*, insula; ic*, internal capsule; IPL*, inferior parietal cortex; LF-WM, lateral frontal WM; (L) PL-WM, (left) parietal WM; Md*, medulla; MFG, medial frontal gyrus; MTG*, middle temporal gyrus; $\mathrm{OL}^{*}$, occipital lobes; OF-WM, orbitofrontal WM; PL-WM, parietal WM; Pons*, pons; $\mathrm{R}^{*}$, red nucleus; R FP-WM, right frontoparietal WM; RLL, right limbic lobe; RR, retrorubal area of midbrain; SmC, sensorimotor cortex, scP*, superior cerebellar peduncle; $\mathrm{SN}^{*}$, substantia nigra; $\mathrm{SPL}^{*}$, superior parietal lobule; Str*, striatum; TL*, temporal WM; Th*, thalamus; VIM, ventral intermediate nucleus of the thalamus; Ver*, vermis. The asterisks denote anatomic abbreviations from Mai et al. ${ }^{54}$

Tangible inconsistencies between different ET neuroimaging studies emerge from the review of existing literature. These need to be addressed to resolve potential neuroimaging markers for this condition. Discrepancies could be due to methodologic differences; but different subjects examined may embody different subtypes of ET. The establishment of neuroimaging consortia in the context of which sequences and hardware could be evaluated and calibrated, as well as effective data-sharing mechanisms, could effectively eliminate site-dependent technical idiosyncrasies. Achieving shared neuroimaging resources based on well-defined (and transparent) protocols has proved very successful, ${ }^{24}$ and it would be especially effective in the case of ET, for which the neuropathogenesis is so elusive.

The confederation of existing and emerging brain banks that share an interest in ET would create the basis for large-scale histologic studies, thereby gradually assembling the complete cellular-level landscape of the disease from thousands of cases as has been done for Alzheimer and Parkinson diseases. This approach would require not only new investments in resources (brain banks are expensive both in terms of operation and real estate) but also active engagement of the public via "citizen science" and partnerships with advocacy groups, the latter often being tremendously successful in educating and mobilizing patient populations (http://www.essentialtremor.org).
Most important, we believe that it is crucial for future research to aim at the correlation of longitudinal neuroimaging data with neuropsychologic testing, quantitative measurements of tremor characteristics (such as frequency and amplitude), and detailed personal medical and biographic information. Our close interactions with (still) few but exceptionally engaged research participants have demonstrated how varied the symptoms can be and the differences in the history of and attitude toward the condition. Quantitative and qualitative differences among patients with ET are often reflected in the results of the complete spectrum of their tests; further studies will determine whether these personal differences are also traceable to distinct patterns in their individual MR imaging. ${ }^{39}$

\section{ACKNOWLEDGMENTS}

The authors would to thank Colleen Sheh for creating the images contained in this manuscript and Clinton and Margaret Spangler for their extraordinary commitment to ET research.

\section{REFERENCES}

1. Louis ED, Ottmann R, Hauser WA. How common is the most common adult movement disorder? Estimates of the prevalence of essential tremor throughout the world. Mov Disord 1998;13:5-10

2. Deuschl G, Bain P, Brin M. Consensus statement of the movement 
disorder society on tremor: Ad Hoc Scientific Committee. Mov Disord 1998;13(suppl 3):2-23

3. Bermejo-Pareja F, Puertas-Martin V. Cognitive features of essential tremor: a review of the clinical aspects and possible mechanistic underpinnings. Tremor Other Hyperkinet Mov (NY) 2012;2 [Epub 2012 Sep 14]

4. Louis ED, Ottman R, Ford B. The Washington Heights-Inwood genetic study of essential tremor: methodological issues in essential tremor research. Neuroepidemiology 1997;16:124-33

5. Braak H, Braak E. Neuropathological staging of Alzheimer-related changes. Acta Neuropathol 1991;82:239-59

6. Braak H, Del Tredici K, Rueb U, et al. Staging of brain pathology related to sporadic Parkinson's disease. Neurobiol Aging 2003; 24:197-211

7. Annese J. The importance of combining MRI and large-scale digital histology in neuroimaging studies of brain connectivity and disease. Front Neuroinform 2012;6:13

8. Cerasa A, Passamonti L, Novellino F, et al. Fronto-parietal overactivation in patients with essential tremor during stroop task. $\mathrm{Neu}$ roreport 2010;21:148-51

9. Passamonti L, Novellino F, Cerasa A, et al. Altered cortical-cerebellar circuits during verbal working memory in essential tremor. Brain 2011;134:2274-86

10. Schmahmann JD, Sherman JC. The cerebellar cognitive affective syndrome. Brain 1998;121:561-79

11. Benito-Leon J, Louis ED, Bermejo-Pareja F. Elderly-onset essential tremor is associated with dementia. Neurology 2006;66:1500-05

12. Louis ED, Zheng W, Jurewicz EC, et al. Elevation of blood $\boldsymbol{\beta}$-carboline alkaloids in essential tremor. Neurology 2002;59:1940-44

13. Higgins JJ, Jankovic J, Patel PI. Evidence that a gene for essential tremor maps to chromosome $\mathbf{2 p}$ in four families. Mov Disord 1998;13:972-77

14. Lorenz D, Deuschl G. Update on pathogenesis and treatment of essential tremor. Curr Opin Neurol 2007;20:447-52

15. Louis ED, Vonsattel JP. The emerging neuropathology of essential tremor. Mov Disord 2008;23:174-82

16. Stewart WF, Schwartz BS, Davatzikos C, et al. Past adult lead exposure is linked to neurodegeneration measured by brain MRI. Neurology 2006;66:1476-84

17. Louis ED. Essential tremor. N Engl J Med 2001;345:887-91

18. Lyons KE, Pahwa R. Thalamic deep brain stimulation and essential tremor. In: Tarsy D, Vitek JL, Star PA, et al, eds. Deep Brain Stimulation in Neurological and Psychiatric Disorders. New York: Humana Press; 2008:205-14

19. Perlmutter JS, Mink JW. Deep brain stimulation. Annu Rev Neurosci 2006;29:229-57

20. Boecker H, Wills AJ, Ceballos-Baumann A, et al. The effect of ethanol on alcohol-responsive essential tremor: a positron emission tomography study. Ann Neurol 1996;39:650-58

21. Gironell A, Kulisevsky J, Lorenzo J, et al. Transcranial magnetic stimulation of the cerebellum in essential tremor. Arch Neurol 2002;59:413-17

22. Louis ED, Faust P, Vonsattel JP, et al. Older onset essential tremor: more rapid progression and more degenerative pathology. Mov Disord 2009;24:1606-12

23. Louis ED, Benito-Leon J, Vega-Quiroga S, et al. Faster rate of cognitive decline in essential tremor cases than controls: a prospective study. Eur J Neurol 2010;17:1291-97

24. Mueller SG, Weiner MW, Thal LJ, et al. The Alzheimer's disease neuroimaging initiative. Neuroimaging Clin N Am 2005;15:869-77, xi-xii

25. Louis ED, Faust PL, Vonsattel JPG, et al. Neuropathological changes in essential tremor: 33 cases compared with 21 controls. Brain 2007;130:3297-307

26. Louis ED. Essential tremor: evolving clinicopathological concepts in an era of intensive post-mortem enquiry. Lancet Neurol 2010;9:613-22
27. Louis ED, Vonsattel JPG, Honig LS, et al. Neuropathologic findings in essential tremor. Neurology 2006;66:1756-59

28. Deuschl G, Elble R. Essential tremor: neurodegenerative or nondegenerative disease towards a working definition of ET. Mov Disord 2009;24:2033-41

29. Erickson-Davis C, Faust PL, Vonsattel J-PG, et al. "Hairy baskets" associated with degenerative Purkinje cell changes in essential tremor. J Neuropathol Exp Neurol 2010;69:262-71

30. Rajput AH, Robinson CA, Rajput ML, et al. Essential tremor is not dependent upon cerebellar Purkinje cell loss. Parkinsonism Relat Disord 2012;18:626-28

31. Shill HA, Adler CH, Sabbagh MN, et al. Pathologic findings in prospectively ascertained essential tremor subjects. Neurology 2008; 70:1452-55

32. Quattrone A, Cerasa A, Messina D, et al. Essential head tremor is associated with cerebellar vermis atrophy: a volumetric and voxelbased morphometric MR imaging study. AJNR Am J Neuroradiol 2008;29:1692-97

33. Cerasa A, Messina D, Nicoletti G, et al. Cerebellar atrophy in essential tremor using an automated segmentation method. AJNR Am J Neuroradiol 2009;30:1240-43

34. Benito-León J, Alvarez-Linera J, Hernandez-Tamames JA, et al. Brain structural changes in essential tremor: voxel-based morphometry at 3-Tesla. J Neurol Sci 2009;287:138-42

35. Bagepally BS, Bhatt MD, Chandran V, et al. Decrease in cerebral and cerebellar gray matter in essential tremor: a voxel-based morphometric analysis under 3T MRI. J Neuroimaging 2012;22:275-78

36. Daniels D, Peller M, Wolff S, et al. Voxel-based morphometry shows no decreases in cerebellar gray matter volume in essential tremor. Neurology 2006;24:1452-56

37. Passamonti L, Cerasa A, Quattrone A. Neuroimaging of essential tremor: what is the evidence for cerebellar involvement? Tremor Other Hyperkinet Mov (N Y) 2012;2 [Epub 2012 Sep 17]

38. Marvel CL, Desmond JE. The contributions of cerebro-cerebellar circuitry to executive verbal working memory. Cortex 2010;46:880-95

39. Klaming R, Kayano J, Bartsch H, et al. Multimodal longitudinal imaging and close monitoring of single-case studies may demonstrate the relationship between structural and behavioral markers of essential tremor. In: Proceedings of the Annual Meeting of the Society for Neuroscience, Washington, DC; November 12-16, 2011

40. Nicoletti G, Manners D, Novellino F, et al. Diffusion tensor MRI changes in cerebellar structures of patients with familial essential tremor. Neurology 2010;74:988-94

41. Klein JC, Lorenz B, Kang JS, et al. Diffusion tensor imaging of white matter involvement in essential tremor. Hum Brain Mapp 2011;32:896-904

42. Saini J, Bagepally BS, Bhatt MD, et al. Diffusion tensor imaging: tract based spatial statistics study in essential tremor. Parkinsonism Relat Disord 2012;18:477-82

43. Martinelli P, Giovanni R, Manners D, et al. Diffusion-weighted imaging study of patients with essential tremor. Mov Disord 2007;22:1182-85

44. Jia L, Jia-Lin S, Qin D, et al. A diffusion tensor imaging study in essential tremor. J Neuroimaging 2011;21:370-74

45. Mori S, Zhang J. Principles of diffusion tensor imaging and its applications to basic neuroscience research. Neuron 2006;51:527-39

46. Shin DH, Han BS, Kim HS, et al. Diffusion tensor imaging in patients with essential tremor. AJNR Am J Neuroradiol 2008;29:151-53

47. Jenkins IH, Bain PG, Colebatch JG, et al. A positron emission tomography study for essential tremor: evidence for overactivity of cerebellar connections. Ann Neurol 1993;34:82-90

48. Wills AJ, Jenkins IH, Thompson PD, et al. Red nuclear and cerebellar but not olivary activation associated with essential tremor: a positron emission tomographic study. Ann Neurol 1994;36:636-42 
49. Bucher SF, Seelos K, Dodel RC, et al. Activation mapping in essential tremor with functional magnetic resonance imaging. Ann Neurol 1997;41:32-40

50. Schnitzler A, Muenks C, Butz M, et al. Synchronized brain network associated with essential tremor as revealed by magnetoencephalography. Mov Disord 2009;24:1629-35

51. Halliday DM, Conway BA, Farmer SF, et al. Coherence between lowfrequency activation of the motor cortex and tremor in patients with essential tremor. Lancet 2000;355:1149-53
52. Louis ED, Shungu DC, Chan S, et al. Metabolic abnormality in the cerebellum in patients with essential tremor: a proton magnetic resonance spectroscopy imaging study. Neurosci Lett 2002;333: 17-20

53. Pagan F, Butman JA, Dambrosia JM, et al. Evaluation of essential tremor with multi-voxel magnetic resonance spectroscopy. Neurology 2003;60:1344-47

54. Mai J, Paxinos G, Voss T. Atlas of the Human Brain. 3rd ed. New York: Elsevier; 2008 